\title{
Non-infectious orbital vasculitides
}

\begin{abstract}
Non-infectious vasculitides comprise a large number of diseases. Many of these diseases can cause inflammation within the orbit and a clinical presentation, which mimics numerous other processes. Orbital disease can often be the initial presentation of a systemic process and early diagnosis can help prevent longterm, potentially fatal consequences. The evaluation and treatment of non-infectious orbital vasculitides are often complicated and require a thorough understanding of the disease and underlying systemic associations. The long-term prognosis visually and systemically must be weighed against the risks and benefits of the treatment regimen. A large variety of corticosteroid formulations currently exist and are the mainstay of initial treatment. Traditional steroid-sparing immunosuppressive agents are also an important arsenal against these vasculitides.

Recently, a new class of drugs called biologics, which target the various mediators of the inflammation cascade, may potentially provide more effective and less toxic treatment. This review aims to synthesize the current literature on non-infectious orbital vasculitides.
\end{abstract}

${ }^{1}$ Kresge Eye Institute, Wayne State University School of Medicine, Detroit, MI, USA

${ }^{2}$ Department of Ophthalmology and Visual Science, Yale University School of Medicine, New Haven, CT, USA

${ }^{3}$ Beaumont Eye Institute, William Beaumont Hospital, Royal Oak, MI, USA

Correspondence: JJ Servat, Beaumont Eye Institute, William Beaumont Hospital, 3535 West 13 Mile Road, Royal Oak, MI 48073, USA Tel: + 12485512020 ;

Fax: + 12485512267

E-mail: javierservat@gmail. com

Received: 8 November 2011 Accepted in revised form:

2 January 2012

Published online:

24 February 2012
Eye (2012) 26, 630-639; doi:10.1038/eye.2012.28; published online 24 February 2012

Keywords: orbital vasculitides; orbital Wegener's granulomatosis; orbital Churg-Strauss; orbital polyarteritis nodosa; orbital Kawasaki; orbital dermatomyositis

\section{Introduction}

Non-infectious, or autoimmune, vasculitides comprise a large number of diseases, and affect a variety of different organ systems. Many of these diseases can cause inflammation within the orbit and cause a clinical presentation, which mimics numerous other processes. It is a spectrum of diseases that can affect all age groups and can lead to significant morbidity and mortality if unrecognized. Orbital disease can often be the initial presentation of a systemic process and early diagnosis can help prevent long-term, potentially fatal consequences. This review aims to synthesize the current literature on non-infectious orbital vasculitides.

\section{Clinical presentation and differential diagnosis}

The clinical presentation of a non-infectious orbital vasculitis is nonspecific and depends on the extent of involvement, which structures are affected, and the specific etiology. Any of the orbital structures can be infected, including orbital fat, orbital nerves, extraocular muscles, lacrimal glands, and the retrobulbar optic nerve. Patients can present with anything from mild ocular pain to severe vision loss. The symptoms and signs are often very similar to orbital inflammatory syndrome (OIS), including erythema and edema of the eyelids and diplopia. Signs of orbital vasculitis include proptosis, an afferent pupillary defect, and limited extraocular muscle movements.

Given the nonspecific nature of presentation for patients with an orbital vasculitis, there is a vast differential diagnosis. The differential can be grouped into three categories: idiopathic inflammatory disorders, neoplasms, and infectious disease (see Table 1). Idiopathic conditions can cause a pseudo-mass lesion in the orbit or cause inflammation of the orbital structures. Oftentimes neoplasms can develop an inflammatory component and cause symptoms/signs such as eyelid erythema and conjunctival injection. Infections secondary to bacteria, viruses, fungi, and parasites are frequently the first consideration in these patients and must always be the differential.

\section{Wegener's granulomatosis}

Wegener's granulomatosis (WG) is the most common non-infectious vasculitis, which can lead to orbital inflammation, and it must be 
Table 1 Differential diagnosis for non-infectious orbital vasculitis

Idiopathic inflammatory Neoplasm

disorders

\begin{tabular}{ll}
\hline Thyroid eye disease & Metastatic disease \\
Sarcoidosis & Rhabdomyosarocma \\
Crohn's disease & Lymphoma \\
Erdheim-Chester & Lymphoproliferative disorders (eg, \\
syndrome & lymphomatoid granulomatosis, \\
Histiocytosis X & angiolymphoid hyperplasia) \\
Scleroderma & Dermoid cyst \\
Sclerosing cholangitis & Lymphangioma \\
Orbital inflammatory & \\
syndrome & \\
& \\
Infectious disease &
\end{tabular}

Bacterial, viral, fungal,

parasitic

considered in patients presenting with orbital signs. It is an autoimmune disease that can affect a number of different organ systems. The pathology of this disease includes necrosis of tissue, granulomatous inflammation, and a vasculitis of small- to medium-sized vessels. These three findings comprise the classic triad of WG, although histological samples exhibit varying degrees of the components. There is a strong association in laboratory studies with anti-neutrophil cytoplasmic antibodies (ANCAs), which can be identified in $80-90 \%$ of cases. ${ }^{1,2}$ These antibodies are thought to be related to the pathogenesis of the disease, although they are not essential. C-ANCA (which reacts with proteinase-3) is more commonly associated with WG than p-ANCA (which reacts with myeloperoxidase), and studies have shown an overall sensitivity of $91 \%$ and specificity of 99\% for c-ANCA. ${ }^{2,3}$ Other laboratory tests are nonspecific and include anemia, thrombocytosis, leukocytosis as well as elevated erythrocyte sedimentation rate (ESR) and C-reactive protein (CRP). There is also evidence that proliferation of Th1 and Th2 lymphocytes contribute to the pathogenesis. WG is a fairly rare entity; the annual incidence is 3 per 100000 . It affects both genders equally and is more common in Caucasians than other races. ${ }^{4}$

The disease most often manifests itself in the lower and upper respiratory tracts $(85 \%)$ as well as the kidneys $(80 \%)$, although ocular involvement is quite common and is seen in $50-60 \%$ of patients with WG. ${ }^{1}$ It can occur as the initial presentation of the disease ( $8-16 \%$ of patients) or as a later sequelae. Orbital involvement is less frequent and has been recognized in $15-20 \%$ of patients. ${ }^{1,5-8}$ Orbital disease can occur de novo, or be secondary to the extension of sinus disease. It can cause significant morbidity to patients and potentially lead to complete vision loss. ${ }^{9}$
In WG, diffuse or focal vascular inflammation within the orbit leads to symptoms of pain, diplopia, and decreased vision, while signs consist of conjunctival injection, proptosis, and ophthalmoplegia. ${ }^{1,8,10-13}$ Diplopia can be due to mass effect on the extraocular muscles, or inflammation and subsequent occlusion of the vessels supplying the muscles. ${ }^{13}$ Vision loss is secondary to optic nerve compression and/or infiltration of the nerve from adjacent orbital disease. ${ }^{14}$ Significant vision loss is a serious consequence of WG and occurs in up to $50 \%$ of patients with orbital involvement. ${ }^{1}$ Inflammation of the lacrimal glands can also be found. In addition, chronic orbital inflammation can eventually lead to fibrosis and socket contracture. This can cause ongoing restriction of extraocular muscle movement and orbital pain. Thus far, this entity has been unresponsive to current treatments for WG. ${ }^{15}$

The diagnosis of orbital disease is ultimately confirmed by orbital biopsy. The histology will demonstrate a vasculitis, granulomatous inflammation, and/or necrosis in $75-85 \%$ of patients. ${ }^{11,16,17}$ Imaging modalities including CT and MRI also help to distinguish Wegener's from other etiologies and confirm the diagnosis. Lesions on CT with contrast appear hyper-intense relative to nasal mucosa. CT can also show obliteration of tissue planes and bony erosion. ${ }^{9,18} \mathrm{On}$ MRI, the lesions are hypo-intense in relation to orbital fat in T1 and T2, and enhance with gadolinium. MRI can assist in specifically identifying granulomatous inflammation as well. ${ }^{9,19}$

Before the use of immunomodulatory medicines for WG the disease was almost always fatal. ${ }^{20}$ In the 1970s, the use of glucocorticoids with cyclophosphamide helped to significantly improve mortality. The current standard of care is a combination of glucocorticoids with cyclophosphamide, methotrexate, or azathioprine.

Survival rates are up to $95 \%$ at 5-year follow-up and $80 \%$ at 10 years. ${ }^{20,21-23}$ More recently, the use of rituximab has been evaluated. Rituximab is an antibody directed against the CD20 surface glycoprotein on B cells. It was thought that this medication may be effective in WG because of the role of ANCA in the pathogenesis, which is produced by B cells. The RAVE study has demonstrated efficacy similar to cyclophosphamide with a better side effect profile. Rituximab does not usually have many of the severe side effects of prednisone and cyclophosphamide such as adrenal suppression, carcinogenicity, and hemorrhagic cystitis. ${ }^{24}$ The treatment of orbital disease is usually identical to the treatment of systemic disease. There has been one report that IV pulse corticosteroids may lead to faster recovery from orbital disease, although this has not been confirmed with subsequent studies. ${ }^{25}$ There have also been encouraging reports on the use of rituximab for 
refractory orbital disease. A case series demonstrated benefit from rituximab use in 10 out of 10 patients tested. In four of these patients, clinical remission was seen for 12 or more months without the need for immunosuppressive medications or steroids. In addition, none of the patients treated had any significant side effects from the rituximab treatment or experienced a serious infection in the period of follow-up. ${ }^{26,27}$ Two other case series have demonstrated benefit from antithymocyte globulin and mycofenolate mofetil in patients, which were refractory to prednisone. ${ }^{28,29} \mathrm{On}$ rare occasion, orbital decompression is necessary to prevent further vision loss in cases of severe disease. ${ }^{8,30}$

\section{Churg-Strauss syndrome}

Another systemic vasculitis that can cause disease within the orbit is Churg-Strauss syndrome (CSS). This is a systemic allergic disease that initially manifests with asthma and allergic rhinitis. In addition, it causes a necrotizing vasculitis of small to medium-sized vessels and granulomatous inflammation, which is rich in eosinophils. ${ }^{31}$

The disease chiefly affects the lungs, sinuses, and peripheral nervous system. In 1990, the American College of Rheumatology established that four out of the following six criteria must be met to make the diagnosis: presence of asthma, hypereosinophilia, mononeuropathy or polyneuropathy, pulmonary infiltrates, paranasal sinus abnormality, and extravascular eosinophil infiltration in biopsy specimens. ${ }^{32}$ Ocular manifestations are rare and they have been divided into two categories: ischemic vasculitis and orbital inflammatory pseudotumor. Signs of an ischemic vasculitis include amaurosis fugax, ischemic optic neuropathy, and retinal artery occlusions. ${ }^{33-35}$ Patients with orbital inflammatory pseudotumor often present with diffuse orbital inflammation, dacryoadenitis, myositis, periscleritis, and perineuritis. Patients can subsequently develop proptosis, diplopia, ophthalmoplegia, and vision loss from optic nerve compression. As CSS and orbital involvement is a relatively rare entity, most information regarding this in the literature is in the form of case reports. ${ }^{36-41}$ For example, Pradeep et $a l^{38}$ described two cases of bilateral orbital inflammation in which both patients presented with proptosis and restriction of extraocular movements.

Diagnosis of orbital involvement secondary to CSS can be confirmed with biopsy of an extraocular muscle, lacrimal gland, or orbital mass. The key on histology to identifying Churg-Strauss is a necrotizing vasculitis with extravascular infiltration of eosinophils. ${ }^{38-40}$ The presence of systemic findings can also help to determine CSS as the cause. Laboratory studies include eosinophilia and positive p-ANCA; $\mathrm{p}$-ANCA has been shown to be present in approximately $70 \%$ of patients with CSS. ${ }^{31}$ $\mathrm{CT}$ and MRI results are less specific and demonstrate enlargement of the retro-orbital space with swelling of the lacrimal glands and extraocular muscles. ${ }^{38,40}$

Treatment of orbital vasculitis secondary to CSS primarily consists of corticosteroids. Cases in the literature have shown an excellent response to oral prednisone with almost complete resolution of orbital inflammation. Methotrexate and cyclophosphamide have also been used in addition to corticosteroids with similar success to prednisone monotherapy. ${ }^{38,40}$ As orbital involvement is fairly infrequent, therapy is almost always in context with treatment of the systemic disease. There have been some successful reports on the use of biologics in refractory or relapsing CSS, although not specifically orbital involvement. Medications used include rituximab, infliximab, and etanercept (antitumor necrosis factor (TNF) antibodies), mepolizumab (anti-interleukin 5 (IL-5) antibody), and omalizumab (anti-IVIG E antibody). These may be helpful options for orbital disease in the future. ${ }^{42}$

\section{Polyarteritis nodosa}

Polyarteritis nodosa (PAN) is a necrotizing vasculitis that chiefly affects medium- and small-sized arteries, and can rarely affect venules. The vasculitis is typically segmental and focal, and can be found in almost any artery of the body. In most patients the etiology cannot be identified, although there has been strong evidence for an association between hepatitis B infection and PAN. It is a disease that is most commonly found in adults aged 40 to 60 years, although it can occur in children and the elderly as well. In the acute phase, histology demonstrates fibrinoid necrosis of the media with an infiltrate consisting mostly of neutrophils; aneurysms and thromboses can occur at the sites of necrosis. When these lesions begin to heal, fibrosis develops that can lead to vessel occlusion. ${ }^{4}$

Similar to other autoimmune vasculitides, PAN affects numerous organ systems and thus the clinical presentation can be varied. It can affect joints/bones, the peripheral nervous system, the central nervous system, kidneys, heart, and gastrointestinal system. ${ }^{4}$ The skin, eye, and orbit are affected in approximately $10 \%$ of patients. Ocular manifestations are usually limited to a retinal vasculitis, ischemic optic neuropathy, or scleritis. ${ }^{43,44}$ As PAN has been identified as a distinct disease, there have been several reports of orbital involvement. PAN can cause ischemia and dysfunction of the extraocular muscles, leading to ophthalmoplegia and diplopia. ${ }^{45}$ PAN can also cause diffuse orbital inflammation, leading to exophthalmos, restriction of 
extraocular movements, conjunctival injection and chemosis, and decreased vision. There have been seven cases in the literature reporting exophthalmos and orbital inflammation associated with PAN. ${ }^{45-48}$ These signs and symptoms are all secondary to the mass effect created by the vasculitis occurring within the orbit.

There is no concrete method for diagnosing orbital disease secondary to PAN. The diagnosis is made when orbital inflammation occurs in association with other signs and symptoms consistent with PAN. Laboratory findings are generally nonspecific and can demonstrate an elevated ESR, eosinophilia, positive anti-nuclear antibody (ANA), and positive rheumatoid factor. ${ }^{4}$ CT and MRI findings are not well defined at this time. There have been isolated reports showing swelling and increased density of the extraocular muscles and soft tissues on CT, and enlargement of muscles and the retrobulbar space on MRI. ${ }^{45,46}$ Orbital biopsies can exhibit a vasculitis of arteries and/or veins with fibrosis, although literature has not supported this as a reliable finding. ${ }^{45,47}$

Treatment of orbital vasculitis secondary to PAN is identical to treatment of the systemic disease. The mainstay of therapy is corticosteroids and cyclophosphamide with case reports showing good response of orbital disease to corticosteroids alone. ${ }^{45,46}$ Other immunomodulatory agents such as azathioprine and methotrexate have also been used. ${ }^{4}$ There have been case reports of disease remission with the use of rituximab in PAN, although not specifically in orbital disease. It may be a treatment to consider when presented with a patient who has orbital inflammation likely secondary to PAN. ${ }^{49}$

\section{Atypical Cogan syndrome}

Cogan syndrome (CS) is characterized by a bilateral nonsyphilitic interstitial keratitis and audiovestibular involvement. One of the systemic manifestations of the disease is a necrotizing vasculitis, which can affect vessels of all sizes. Some of the more common vessels involved are the aorta, renal arteries, and coronary arteries. ${ }^{50,51}$ Atypical CS (ACS) was defined in 1980 by Haynes et al ${ }^{52}$ it encompasses CS along with other ocular manifestations including orbital inflammation, scleritis, retinitis, glaucoma, artery occlusions, and optic neuritis. CS and ACS classically occurs in young adults and equally in males and females. ${ }^{51}$

The patients will typically develop vertigo, imbalance, and hearing loss in addition to blurred vision, conjunctival injection, and eye pain. Oftentimes there is a 2-year delay between the presentation of ocular and audiovestibular symptoms. ${ }^{51}$ Orbital involvement is uncommon; there have been four cases reported in the literature. These patients presented with hearing loss and vertigo along with exophthalmos and orbital inflammation. CT demonstrated evidence for myositis as well. None of these patients underwent an orbital biopsy and therefore it is difficult to determine if the inflammation was truly secondary to a vasculitis. ${ }^{53-55}$ Treatment of ACS consists of a glucocorticoid and occasionally an immunodulatory agent. ${ }^{51}$ Patients with orbital inflammation described in the literature were successfully treated with a combination of prednisone and cyclophosphamide, ${ }^{53-55}$ although given the scarcity of cases it is uncertain if cyclophosphamide is a necessary addition to the treatment regimen.

\section{Temporal arteritis}

Temporal arteritis (TA), also known as giant cell arteritis or Horton's disease, is an idiopathic vasculitis of medium to large caliber arteries of the head and neck region. The branches coming off the carotid artery are most often affected. ${ }^{56}$ It is a disease that is seen mostly in Caucasians over 50 years of age.$^{57}$ Classically, the histology demonstrates segmental inflammation of arteries consisting of giant cells, lymphocytes, plasma cells, and eosinophils in the lamina media. There is also necrosis of the smooth muscle and thickening of the internal elastic lamina. ${ }^{58}$ Laboratory studies often reveal an elevated ESR and CRP, although $20 \%$ of patients with TA will have a normal ESR. ${ }^{59}$ The American College of Rheumatology published diagnostic criteria in 1990 and TA can be diagnosed if three of the following are present: age $>50$, new headache, temporal artery abnormality, elevated ESR, or abnormal temporal artery biopsy. ${ }^{60}$

The most common symptoms are a unilateral throbbing temporal headache, scalp tenderness, fever, and fatigue. ${ }^{61,62}$ In addition, patients can have stiffness, neck and shoulder pain, and jaw claudication. ${ }^{56}$ The classic ophthalmological symptom is vision loss, which can be preceded by episodes of amaurosis fugax. This is secondary to ischemia of the optic nerve or retinal artery occlusion. ${ }^{63,64}$

TA can also cause symptoms secondary to ischemia and inflammation within the orbit. It can manifest as an orbital infarction syndrome and lead to ophthalmoplegia and diplopia, in addition to vision loss from arterial occlusion. ${ }^{65}$ Ophthalmoplegia and diplopia can also occur separately due to occlusion of arteries supplying the extraocular muscles. ${ }^{64}$ A less common presentation of TA is orbital inflammation; these patients can have many of the same signs and symptoms as OIS, which include proptosis, chemosis, and conjunctival injection. They can also have diplopia, vision loss, and an afferent pupillary defect secondary to mass effect on the extraocular muscles and the optic nerve. ${ }^{6-68}$ 
The diagnosis of TA orbital disease can be determined from the combination of symptoms/signs, laboratory studies, radiological imaging, and temporal artery biopsy. Patients with orbital signs secondary to TA will also frequently have a unilateral temporal headache, scalp tenderness, as well as other classic symptoms of TA. The ESR is typically elevated and the temporal artery biopsy confirms the histological signs mentioned earlier for TA. ${ }^{66-68}$ There is no well-defined appearance on imaging, although MRI can show contrast enhancement of the orbit with an infiltrative appearance or a mass lesion. ${ }^{66,68}$ On rare occasion an orbital biopsy is necessary to confirm the diagnosis; this can reveal giant cells, loss of the elastic lamina of arteries, and areas of fibrosis and necrosis. ${ }^{66,68}$

Currently, the treatment of orbital vasculitis secondary to TA is no different than treatment without orbital disease. Patients are immediately started on high-dose corticosteroids, which are slowly tapered after an initial response. There have been various alternative treatments, which have been used as well. Cyclophosphamide has shown to be beneficial in cases that are refractory to steroids. ${ }^{68}$ In addition, Cockerham et al ${ }^{66}$ reported one case of orbital inflammation associated with TA, which was responsive to external beam radiation. This is an option that is reserved for the most severe cases, which are refractory to steroids. Treatment of TA with biologic agents such as anti-TNF alpha antibodies has had mixed success. Recently, treatment with tocilizumab (anti-IL-6 antibody) has been reported in isolated cases. The use of these medications in orbital disease is yet to be tested. ${ }^{69}$

\section{Kawasaki syndrome}

Unlike most of the etiologies discussed in this review, Kawasaki's disease (KD) is a systemic autoimmune vasculitis, which occurs mostly in children $<5$ years of age. The vasculitis predominantly affects the coronary arteries, although other vessels (such as the subclavian, renal, iliac, mesenteric, and digital arteries) can be involved as well. ${ }^{4,70}$ Although originally described in Japan, the disease is seen in children worldwide and in all races. There are six characteristic lesions seen in this vasculitis: endothelial degeneration, necrotizing arteritis, granulomatous inflammation, degeneration of the media, scar formation, and aneurysm formation. Laboratory studies are nonspecific, and can demonstrate an elevated ESR, CRP, alpha1-antitrypsin levels, and platelet counts. ${ }^{4}$

The diagnosis of KD is made with the presence of six cardinal symptoms: fever persisting $>5$ days, erythema and induration of the palms and soles with desquamation in the later stages, polymorphous exanthema, bilateral conjunctival chemosis and injection, erythema of oral mucosa, and acute cervical lymphadenopathy. The other key feature of $\mathrm{KD}$ is the development of coronary aneurysms; $30 \%$ of untreated patients will develop an aneurysm. ${ }^{4}$

The ocular components characteristically associated with KD are conjunctival injection and an anterior uveitis. ${ }^{4}$ To date, there have been three case reports of orbital involvement in association with KD. ${ }^{70-72}$ Felz et $a l^{70}$ described a child with classic symptoms of KD who developed unilateral lid edema, hypoglobus, and impaired upgaze, and was found to have orbital inflammation. Sheard et $a l^{72}$ and Demirsoy et $a l^{71}$ also described cases of orbital involvement that was diagnosed with $\mathrm{CT} .{ }^{70}$ Orbital exploration and biopsy was performed on one patient, which demonstrated edematous orbital fat with a pale superior rectus and attenuation of ciliary vessels. An orbicularis muscle biopsy in the same patient indicated a panarteritis. ${ }^{70}$

The standard of care for management of KD is high-dose IVIG with aspirin, which works to decrease the duration of symptoms as well as prevent the development of coronary artery aneurysms. The use of corticosteroids in KD is controversial, and is most often used in refractory disease. Two of the patients mentioned previously with orbital disease improved with use of IVIG and aspirin alone, and the other patient improved with intravenous methylprednisolone..$^{70-72}$ Recently, infliximab has been used as an initial treatment for $\mathrm{KD}$ and in refractory disease, although it has not been reported in orbital involvement at this time. It may be a good alternative to corticosteroids. ${ }^{73}$ Orbital involvement in $\mathrm{KD}$ is a rare entity that should be considered in the context of other signs and symptoms consistent with the disease.

\section{Behcet's disease}

Behcet's Disease (BD) is another idiopathic systemic vasculitis that affects small- to medium-sized vessels and can involve a number of different organ systems. Although the disease is quite rare in the United States, it is much more common in the Middle East and Asia. Worldwide, males are more commonly affected than females. The pathology is defined by a vasculitis with a lymphocytic and neutrophilic infiltrate; neutrophils and fibrin can enter the vessel lumen leading to thrombosis and occlusion of the vessel. The main laboratory study associated with BD is HLA-B51. Other laboratory studies, such as increased ESR and CRP, are less specific to this disease. $^{4}$

The International Study Group for Behcet's Disease has established a set of guidelines for the diagnosis of BD. Patients must have oral (aphthous) ulcers along with two out of four of the following: genital ulcers, skin 
lesions, eye inflammation (uveitis, vasculitis), or a pathergy reaction. ${ }^{74}$ The hallmark of $\mathrm{BD}$ is aphthous ulcers, although genital ulcers can also be found. Other systemic manifestations occur in the gastrointestinal tract, lungs, muscles, joints, and the brain. Patients can develop severe complications leading to hemoptysis, arthralgias, and dural sinus thrombosis. Ocular involvement is common in BD, manifesting most frequently as a uveitis or retinal vasculitis. BD rarely causes an anterior ischemic optic neuropathy and even less frequently results in orbital involvement. There are three case reports in the literature describing orbital inflammation associated with BD. All three describe inflammation of extraocular muscles and in one case the patient had dacryoadenitis as well. ${ }^{75-77}$ None of these patients had orbital biopsies performed. Given the paucity of data describing BD and orbital involvement and the lack of orbital biopsies, it is difficult to determine if the orbital inflammation was truly secondary to BD. CT and MRI scans demonstrated enhancement and enlargement of extraocular muscles and the lacrimal gland. ${ }^{75-77}$ Diagnosis at this time is made on the association of symptoms, signs, and laboratory studies consistent with BD and the development of orbital inflammation. There have been several studies indicating decreased blood flow in the ophthalmic artery in BD, though it is uncertain if this is related to orbital involvement. $^{78}$

One of the patients reported in the literature with BD and orbital disease was successfully treated with methylprednisolone and then oral prednisone. ${ }^{77}$ Another had resolution of inflammation with prednisone and cyclophosphamide. ${ }^{75}$ The use of infliximab (anti-TNF alpha antibody) in BD has gained popularity and it has been shown to have good success, along with adalimumab and etanercept. ${ }^{79}$ Garrity et $a l^{76}$ reported a patient with poor response to steroids and then improvement of signs and symptoms after the use of infliximab. This is certainly a therapy to consider in a patient with orbital inflammation, which may be secondary to $\mathrm{BD}$.

\section{Systemic lupus erythematosis and rheumatoid arthritis}

Systemic lupus erythematosis (SLE) and rheumatoid arthritis (RA) are both connective tissue diseases, which can have a related vasculitis. Vascular inflammation is a common finding in these patients with an incidence of 30-40\% in SLE and 5-15\% in RA. Both diseases most often affect small vessels with a leukocytoclastic vasculitis. SLE can occasionally involve the great vessels, and RA can lead to necrosis of larger vessels as well. Another similarity between the two entities is the pathogenesis of the vasculitis. Both are thought to be related to immune complex deposition within the vessels walls. There is also some thought that ANCAs may contribute to the vascular inflammation in RA, although there is currently no consensus regarding this aspect of the pathogenesis. ${ }^{4}$

In RA and SLE, the most commonly seen manifestations of a vasculitis are cutaneous lesions (such as nail-fold infarcts, palpable purpura, and digital gangrene) and polyneuropathy. Other areas that can be involved include the mesenteric and coronary arteries, lower extremities, central nervous system, and the pulmonary arteries. The most common ocular findings in both of these diseases are retinopathy, keratoconjunctivitis sicca, and uveitis. ${ }^{4,80}$

Orbital involvement is infrequent in both SLE and RA, although it is a more well-known entity in SLE. There have been a number of case reports in the literature describing diffuse orbital inflammation, orbital infarction, and myositis in association with SLE. In most of these cases, patients had an elevated ANA and dsDNA along with other systemic symptoms consistent with SLE ${ }^{81-91}$ Arthurs et al ${ }^{81}$ described a case of a patient who developed exophthalmos secondary to an orbital mass; biopsy of the mass demonstrated evidence of a pseudotumor with vascular inflammation. This patient eventually developed infarction and subsequent disintegration of orbital structures. Other patients developed a myositis with enlargement of the muscles on CT. ${ }^{83,84,86}$ Reported cases of orbital inflammation in RA mostly involve patients with limited extraocular movements secondary to a myositis. Nabili et al ${ }^{92}$ and Panfilio et $a l^{93}$ reported cases of extraocular muscle enlargement in patients with a prior history of RA. Orbital biopsy was not performed in either case, therefore it is difficult to determine the exact etiology of the myositis.

Similar to other systemic vasculitides that involve the orbit, there is no consensus on treatment. Patients in the literature have been successfully treated with oral or IV glucocorticoids. ${ }^{81-93}$ Most patients had complete resolution of symptoms after initiating therapy, although there were four cases in which the patients had a recurrence of disease and needed an increase in dosage. ${ }^{89-91}$ Treatment with steroids was always in addition to their ongoing treatment for SLE or RA. One patient was also treated with azathioprine. ${ }^{89}$ Other immunomodulatory agents may also have a role in treatment of orbital involvement, although this is yet to be determined. Thus far, the use of biologics for SLE has been controversial, and thus more studies may be necessary before use in orbital disease. Rituximab and infliximab have demonstrated mixed results. Belimumab (anti-B-lymphocyte stimulator) has been beneficial in a phase 3 trial. Infliximab and rituximab have been used 
with much more success in RA, and should certainly be considered as an adjunct therapy in orbital disease. ${ }^{94-96}$

\section{Dermatomyositis}

Dermatomyositis (DM) is an inflammatory myopathy of children and adults; the pathogenesis of this disease is related to a complement mediated microangiopathy. It is an autoimmune disease in which antibodies are directed toward the vascular endothelium, leading to activation of the complement system. The classic laboratory study that helps to diagnose a patient with DM is an elevated creatine kinase level. Electromyography demonstrates increased spontaneous activity and short duration, low amplitude units on voluntary activity. ${ }^{97}$

The main symptom is proximal muscle weakness, which has a subacute course and develops over weeks to months. Patients with DM also develop a rash on the hands, eyelids ('heliotrope'), back, and/or chest. ${ }^{97}$ There have been two case reports of extraocular muscle involvement, which may be associated with DM. Kokotis et $a l^{98}$ reported a case in which a patient with a history of muscle weakness developed bilateral exophthalmos. Laboratory studies were normal except for a mild leukocytosis and CRP. MRI of the orbits revealed enlargement of the lateral and inferior rectus of both eyes and the superior rectus of the left eye. Electromyography demonstrated changes consistent with a myopathy in the frontalis and orbicularis muscles. In a reply to the article by Kokotis et al ${ }^{98}$ Marasini et al ${ }^{99}$ reported a similar case in which a patient developed orbital myositis years after a presentation of DM. The definitive method for diagnosing DM is biopsy of the affected muscle, ${ }^{97}$ although this was not done in either case reported.

Treatment of DM mainly consists of glucocorticoids, although IVIG has been used in some cases as a first-line agent. Other immunosuppressants such as methotrexate and azathioprine have been used as well. ${ }^{97}$ The two patients reported to have orbital involvement improved with the combination of methotrexate and prednisone..$^{98,99}$ The two cases reported in the literature demonstrate possible orbital involvement, although there was no definitive evidence of the association between $\mathrm{DM}$ and orbital myositis. Results of infliximab treatment for DM have not been promising, although rituximab may be a better option. New anti-interferon alpha antibodies may be another option, although studies are ongoing. The efficacy in orbital disease is unknown thus far. ${ }^{100}$

\section{Summary and Conclusions}

Orbital vasculitis is an entity that primarily presents with signs and symptoms of OIS. Most commonly, patients present with exophthalmos, limitation of extraocular movements, conjunctival injection, and/or decreased vision. It can also present with infarction of orbital structures. There are a number of different non-infectious systemic diseases that can be associated with orbital involvement. Although the association between a systemic autoimmune vasculitis and orbital inflammation can be rare, over the past 15 years there has been growing evidence of a relationship between the two.

The evaluation and treatment of non-infectious orbital vasculitides are often complicated and require a thorough understanding of the disease and underlying systemic associations. The long-term prognosis visually and systemically must be weighed against the risks and benefits of the treatment regimen.

A large variety of corticosteroid formulations currently exist and are the mainstay of initial treatment. Traditional steroid-sparing immunosuppressive agents are also an important arsenal against these vasculitides.

Recently, a new class of drugs called biologics, which target the various mediators of the inflammation cascade, may potentially provide more effective and less toxic treatment. The results of biologics for the treatment of systemic rheumatic diseases have led to its off-label use in the treatment of ocular and orbital inflammation. Biologics in general, offer a safer profile compared with conventional immunosuppressive therapies, but clinical experience has demonstrated that they can have unexpected effects, including the risk of tuberculosis activation, invasive fungal, and other opportunistic infections and increase risk of malignancies.

When evaluating a patient with orbital signs, it is important to consider an autoimmune vasculitis as a cause for orbital inflammation or infarction in order to determine the appropriate therapy and potentially diagnose a systemic illness.

\section{Method of literature search}

Articles were searched for in PubMed using different combinations of the following terms: orbit, eye, or ocular with Wegener's granulomatosis, Churg-Strauss, polyarteritis nodosa, systemis lupus erythematosis, atypical Cogan's, temporal arteritis, Kawasaki, dermatomyositis, rheumatoid arthritis, or Behcet's. A separate search was also performed for orbital vasculitis. The resultant articles were evaluated and those with information relevant to vascular inflammation within the orbit were included. Relevant articles listed in bibliographies of these articles were also included. In addition, other selected articles and books with information on the pathogenesis, epidemiology, 
diagnosis, and treatment of the systemic vasculitides were included.

\section{Conflict of interest}

The authors declare no conflict of interest.

\section{References}

1 Hoffman GS, Kerr GS, Leavitt RY, Hallahan CW, Lebovics RS, Travis WD et al. Wegener granulomatosis: an analysis of 158 patients. Ann Intern Med 1992; 116: 488-498.

2 Rao JK, Weinberger M, Oddone EZ, Allen NB, Landsman $\mathrm{P}$, Feussner JR. The role of antineutrophil cytoplasmic antibody (c-ANCA) testing in the diagnosis of Wegener granulomatosis. A literature review and meta-analysis. Ann Intern Med 1995; 123: 925-932.

3 Falk RJ, Jennette JC. Anti-neutrophil cytoplasmic autoantibodies with specificity for myeloperoxidase in patients with systemic vasculitis and idiopathic necrotizing and crescentic glomerulonephritis. N Engl J Med 1988; 318: 1651-1657.

4 Ball GV, Bridges SL. Vasculitis. Oxford University Press: Oxford, 2002.

5 Anderson G, Coles ET, Crane M, Douglas AC, Gibbs AR, Geddes DM et al. Wegener's granuloma. A series of 265 British cases seen between 1975 and 1985. A report by a sub-committee of the British Thoracic Society Research Committee. Q J Med 1992; 83: 427-438.

6 Fauci AS, Haynes BF, Katz P, Wolff SM. Wegener's granulomatosis: prospective clinical and therapeutic experience with 85 patients for 21 years. Ann Intern Med 1983; 98: 76-85.

7 Reinhold-Keller E, Beuge N, Latza U, de Groot K, Rudert H, Nölle B et al. An interdisciplinary approach to the care of patients with Wegener's granulomatosis: long-term outcome in 155 patients. Arthritis Rheum 2000; 43: 1021-1032.

8 Thorne JE, Jabs DA. Ocular manifestations of vasculitis. Rheum Dis Clin North Am 2001; 27: 761-779.

9 Provenzale JM, Mukherji S, Allen NB, Castillo M, Weber AW. Orbital involvement by Wegener's granulomatosis: imaging findings. AJR Am J Roentgenol 1996; 166: 929-934.

10 Haynes BF, Fishman ML, Fauci AS, Wolff SM. The ocular manifestations of Wegener's granulomatosis. Fifteen years experience and review of the literature. Am J Med 1977; 63: 131-141.

11 Perry SR, Rootman J, White VA. The clinical and pathologic constellation of Wegener granulomatosis of the orbit. Ophthalmology 1997; 104: 683-694.

12 Stavrou P, Deutsch J, Rene C, Laws DE, Luqmani RA, Murray PI. Ocular manifestations of classical and limited Wegener's granulomatosis. Q J Med 1993; 86: 719-725.

13 Woo TL, Francis IC, Wilcsek GA, Coroneo MT, McNab AA, Sullivan TJ et al. Australasian orbital and adnexal Wegener's granulomatosis. Ophthalmology 2001; 108: 1535-1543.

14 Lovelace K, Cannon TC, Flynn S, Davis P, Schmucker T, Westfall CT. Optic neuropathy in patient with Wegener's granulomatosis. J Ark Med Soc 2004; 100: 428-429.

15 Talar-Williams C, Sneller MC, Langford CA, Smith JA, Cox TA, Robinson MR. Orbital socket contracture: a complication of inflammatory orbital disease in patients with Wegener's granulomatosis. Br J Ophthalmol 2005; 89: 493-497.

16 Fechner FP, Faquin WC, Pilch BZ. Wegener's granulomatosis of the orbit: a clinicopathological study of 15 patients. Laryngoscope 2002; 112: 1945-1950.

17 Kalina PH, Lie JT, Campbell RJ, Garrity JA. Diagnostic value and limitations of orbital biopsy in Wegener's granulomatosis. Ophthalmology 1992; 99: 120-124.

18 Reuter M, Biederer J, Both M, Schnabel A, Reinhold-Keller E, Gross WL et al. Radiology of the primary systemic vasculitides. Rofo 2003; 175: 1184-1192.

19 Muhle C, Reinhold-Keller E, Richter C, Duncker G, Beigel A, Brinkmann G. MRI of the nasal cavity, the paranasal sinuses and orbits in Wegener's granulomatosis. Eur Radiol 1997; 7: 566-570.

20 Langford CA. Treatment of ANCA-associated vasculitis. N Engl J Med 2003; 349: 3-4.

21 Novack SN, Pearson CM. Cyclophosphamide therapy in Wegener's granulomatosis. N Engl J Med 1971; 284: 938-942.

22 Pagnoux C, Mahr A, Hamidou MA, Boffa JJ, Ruivard M, Ducroix JP et al. Azathioprine or methotrexate maintenance for ANCA-associated vasculitis. N Engl J Med 2008; 359: 2790-2803.

23 Villa-Forte A, Clark TM, Gomes M, Carey J, Mascha E, Karafa MT et al. Substitution of methotrexate for cyclophosphamide in Wegener granulomatosis: a 12-year single-practice experience. Medicine (Baltimore) 2007; 86: 269-277.

24 Stone JH, Merkel PA, Spiera R, Seo P, Langford CA, Hoffman GS et al. Rituximab versus cyclophosphamide for ANCA-associated vasculitis. N Engl J Med 2010; 363: 221-232.

25 Alloway JA, Cupps TR. High dose methylprednisolone for retroorbital Wegener's granulomatosis. J Rheumatol 1993; 20: $752-754$.

26 Avshovich N, Boulman N, Slobodin G, Zeina AR, Rosner I, Rozenbaum M. Refractory Wegener's granulomatosis: effect of rituximab on granulomatous bilateral orbital involvement. Isr Med Assoc J 2009; 11: 566-568.

27 Taylor SR, Salama AD, Joshi L, Pusey CD, Lightman SL. Rituximab is effective in the treatment of refractory ophthalmic Wegener's granulomatosis. Arthritis Rheum 2009; 60: 1540-1547.

28 Kool J, de Keizer RJ, Siegert CE. Antithymocyte globulin treatment of orbital Wegener granulomatosis: a follow-up study. Am J Ophthalmol 1999; 127: 738-739.

29 Yalcindag FN, Amer R, Forrester JV. Mycophenolate mofetil in the treatment of ocular inflammation in ANCA-associated vasculitis. J Ocul Pharmacol Ther 2008; 24: 249-254.

30 Knoch DW, Lucarelli MJ, Dortzbach RK, Smith ME. Limited Wegener granulomatosis with 40 years of followup. Arch Ophthalmol 2003; 121: 1640-1642.

31 Cottin V, Cordier JF. Churg-Strauss syndrome. Allergy 1999; 54: 535-551.

32 Masi AT, Hunder GG, Lie JT, Michel BA, Bloch DA, Arend WP et al. The American College of Rheumatology 1990 criteria for the classification of Churg-Strauss syndrome (allergic granulomatosis and angiitis). Arthritis Rheum 1990; 33: 1094-1100.

33 Acheson JF, Cockerell OC, Bentley CR, Sanders MD. Churg-Strauss vasculitis presenting with severe visual loss 
due to bilateral sequential optic neuropathy. Br J Ophthalmol 1993; 77: 118-119.

34 Alberts AR, Lasonde R, Ackerman KR, Chartash EK, Susin M, Furie RA. Reversible monocular blindness complicating Churg-Strauss syndrome. J Rheumatol 1994; 21: 363-365.

35 Dagi LR, Currie J. Branch retinal artery occlusion in the Churg-Strauss syndrome. J Clin Neuroophthalmol 1985; 5: 229-237.

36 Bosch-Gil JA, Falgà-Tirado C, Simeón-Aznar CP, Orriols-Martínez R. Churg-Strauss syndrome with inflammatory orbital pseudotumour. Br J Rheumatol 1995; 34: $485-486$.

37 Nissim F, Von der Valde J, Czernobilsky B. A limited form of Churg-Strauss syndrome: ocular and cutaneous manifestations. Arch Pathol Lab Med 1982; 106: 305-307.

38 Pradeep TG, Prabhakaran VC, McNab A, Dodd T, Selva D. Diffuse bilateral orbital inflammation in Churg-Strauss syndrome. Ophthal Plast Reconstr Surg 2010; 26: 57-59.

39 Sale S, Patterson R. Recurrent Churg-Strauss vasculitis. With exophthalmos, hearing loss, nasal obstruction, amyloid deposits, hyperimmunoglobulinemia E, and circulating immune complexes. Arch Intern Med 1981; 141: 1363-1365.

40 Takanashi T, Uchida S, Arita M, Okada M, Kashii S. Orbital inflammatory pseudotumor and ischemic vasculitis in Churg-Strauss syndrome: report of two cases and review of the literature. Ophthalmology 2001; 108: 1129-1133.

41 Billing K, Malhotra R, Selva D, Dodd T. Orbital myositis in Churg-Strauss syndrome. Arch Ophthalmol 2004; 122: 393-396.

42 Baldini C, Talarico R, Della Rossa A, Bombardieri S. Clinical manifestations and treatment of Churg-Strauss syndrome. Rheum Dis Clin North Am 2010; 36: 527-543.

43 Cohen RD, Conn DL, Ilstrup DM. Clinical features, prognosis, and response to treatment in polyarteritis. Mayo Clin Proc 1980; 55: 146-155.

44 Hutchinson $\mathrm{CH}$. Polyarteritis nodosa presenting as posterior ischaemic optic neuropathy. J R Soc Med 1984; 77: 1043-1046.

45 Ishida K, Yokota T, Wada Y, Yamada M, Tsukagoshi H. Unilateral facial swelling and exophthalmos in a patient with polyarteritis nodosa. Intern Med 1992; 31: 500-503.

46 Koike R, Yamada M, Matsunaga T, Yoshida S, Tsukagoshi H. Polyarteritis nodosa (PN) complicated with unilateral exophthalmos. Intern Med 1993; 32: 232-236.

47 Vanwien S, Merz EH. Exophthalmos secondary to periarteritis nodosa. Am J Ophthalmol 1963; 56: 204-208.

48 Walton EW. Pseudo tumour of the orbit and polyarteritis nodosa. J Clin Pathol 1959; 12: 419-426.

49 Ribeiro E, Cressend T, Duffau P, Grenouillet-Delacre M, Rouanet-Lariviere M, Vital A et al. Rituxamab efficacy during a refractory polyarteritis nodosa flare. Case Report Med 2009; 2009, doi:10.1155/2009/738293.

50 Chen SI, Sahni J, Hart IK. Atypical Cogan's syndrome presenting with bilateral acute glaucoma. Eye (Lond) 2004 18: 328-329.

51 Grasland A, Pouchot J, Hachulla E, Blétry O, Papo T, Vinceneux $\mathrm{P}$ et al. Typical and atypical Cogan's syndrome: 32 cases and review of the literature. Rheumatology (Oxford) 2004; 43: 1007-1015.

52 Haynes BF, Kaiser-Kupfer MI, Mason P, Fauci AS. Cogan syndrome: studies in thirteen patients, long-term follow-up, and a review of the literature. Medicine (Baltimore) 1980; 59: 426-441.

53 Balayre S, Gicquel JJ, Mercie M, Dighiero P. [Atypical Cogan syndrome]. J Fr Ophtalmol 2003; 26: 64-68.

54 Cobo LM, Haynes BF. Early corneal findings in Cogan's syndrome. Ophthalmology 1984; 91: 903-907.

55 Gran JT, Nordvag BY, Storesund B. An overlap syndrome with features of atypical Cogan syndrome and Wegener's granulomatosis. Scand I Rheumatol 1999; 28: 62-64.

56 Salvarani C, Cantini F, Boiardi L, Hunder GG. Polymyalgia rheumatica and giant-cell arteritis. N Engl J Med 2002; 347: 261-271.

57 Nordborg E, Bengtsson BA. Epidemiology of biopsy-proven giant cell arteritis (GCA). J Intern Med 1990; 227: 233-236.

58 Miller DV, Maleszewski JJ. The pathology of large-vessel vasculitides. Clin Exp Rheumatol 2011; 29(1 Suppl 64): S92-S98.

59 Wise CM, Agudelo CA, Chmelewski WL, McKnight KM. Temporal arteritis with low erythrocyte sedimentation rate: a review of five cases. Arthritis Rheum 1991; 34: 1571-1574.

60 Hunder GG, Bloch DA, Michel BA, Stevens MB, Arend WP, Calabrese LH et al. The American College of Rheumatology 1990 criteria for the classification of giant cell arteritis. Arthritis Rheum 1990; 33: 1122-1128.

61 Calamia KT, Hunder GG. Giant cell arteritis (temporal arteritis) presenting as fever of undetermined origin. Arthritis Rheum 1981; 24: 1414-1418.

62 Solomon S, Cappa KG. The headache of temporal arteritis. J Am Geriatr Soc 1987; 35: 163-165.

63 Bhatti MT, Tabandeh H. Giant cell arteritis: diagnosis and management. Curr Opin Ophthalmol 2001; 12: 393-399.

64 Hayreh SS, Podhajsky PA, Zimmerman B. Ocular manifestations of giant cell arteritis. Am J Ophthalmol 1998; 125: 509-520.

65 Borruat FX, Bogousslavsky J, Uffer S, Klainguti G, Schatz NJ. Orbital infarction syndrome. Ophthalmology 1993; 100: 562-568.

66 Cockerham KP, Cockerham GC, Brown HG, Hidayat AA. Radiosensitive orbital inflammation associated with temporal arteritis. J Neuroophthalmol 2003; 23: 117-121.

67 Fa'Amatuainu NA, Ng J, Kumar S, Green G. Unusual presentations of giant cell arteritis (temporal arteritis). Intern Med J 2004; 34: 654-655.

68 Lee AG, Tang RA, Feldon SE, Pless M, Schiffman JS, Rubin RM et al. Orbital presentations of giant cell arteritis. Graefes Arch Clin Exp Ophthalmol 2001; 239: 509-513.

69 Beyer C, Axmann R, Sahinbegovic E, Distler JH, Manger B, Schett $\mathrm{G}$ et al. Anti-interleukin 6 receptor therapy as rescue treatment for giant cell arteritis. Ann Rheum Dis 2011; 70: 1874-1875.

70 Felz MW, Patni A, Brooks SE, Tesser RA. Periorbital vasculitis complicating Kawasaki syndrome in an infant. Pediatrics 1998; 101: E9.

71 Demirsoy S, Gucuyener K, Olgunturk R, Oguz D, Or M. A case of Kawasaki syndrome associated with preseptal cellulitis in orbita. Turk J Pediatr 1988; 30: 55-59.

72 Sheard RM, Pandey KR, Barnes ND, Vivian AJ. Kawasaki disease presenting as orbital cellulitis. J Pediatr Ophthalmol Strabismus 2000; 37: 123-125.

73 Son MB, Gauvreau K, Burns JC, Cornaldesi E, Tremoulet $\mathrm{AH}$, Watson VE et al. Infliximab for intravenous immunoglobulin resistance in Kawasaki disease: a retrospective study. J Pediatr 2011; 158: 644-649. 
74 Criteria for diagnosis of Behcet's disease. International Study Group for Behcet's Disease. Lancet 1990; 335: 1078-1080.

75 Dursun D, Akova Y, Yucel E. Myositis and scleritis associated with Behcet's disease: an atypical presentation. Ocul Immunol Inflamm 2004; 12: 329-332.

76 Garrity JA, Coleman AW, Matteson EL, Eggenberger ER, Waitzman DM. Treatment of recalcitrant idiopathic orbital inflammation (chronic orbital myositis) with infliximab. Am J Ophthalmol 2004; 138: 925-930.

77 Hammami S, Yahia SB, Mahjoub S, Khairallah M. Orbital inflammation associated with Behcet's disease. Clin Experiment Ophthalmol 2006; 34: 188-190.

78 Isik C, Yagci B, Yildirim C, Yaylali V, Tatlipinar S, Ozden S. Orbital color Doppler imaging in Behcet's disease with or without ocular involvement. Int Ophthalmol 2007; 27: $37-42$

79 Sfikakis PP, Markomichelakis N, Alpsoy E, Assaad-Khalil $\mathrm{S}$, Bodaghi B, Gul A et al. Anti-TNF therapy in the management of Behcet's disease - review and basis for recommendations. Rheumatology (Oxford) 2007; 46: 736-741.

80 Read RW. Clinical mini-review: systemic lupus erythematosus and the eye. Ocul Immunol Inflamm 2004; 12: 87-99.

81 Arthurs BP, Khalil MK, Chagnon F, Lindley SK, Anderson DP, Burnier Jr M. Orbital infarction and melting in a patient with systemic lupus erythematosus. Ophthalmology 1999; 106: 2387-2390.

82 Feinfield RE, Hesse RJ, Rosenberg SA. Orbital inflammatory disease associated with systemic lupus erythematosus. South Med J 1991; 84: 98-99.

83 Keane JR. Eye movement abnormalities in systemic lupus erythematosus. Arch Neurol 1995; 52: 1145-1149.

84 McDonald E, Marino C, Cimponeriu D. Painful ophthalmoplegia in a patient with SLE. Hosp Pract (Off Ed) 1992; 27: 41-44.

85 Sanchez IJ, Finger DR, Bradshaw DJ. Orbital inflammatory syndrome and systemic lupus erythematosus. J Clin Rheumatol 1995; 1: 295-298.

86 Serop S, Vianna RN, Claeys M, De Laey JJ. Orbital myositis secondary to systemic lupus erythematosus. Acta Ophthalmol (Copenh) 1994; 72: 520-523.
87 Siebert S, Srinivasan U. Proptosis can be the presenting feature of systemic lupus erythematosus. Ann Rheum Dis 2004; 63: 908-909.

88 Stavrou P, Murray PI, Batta K, Gordon C. Acute ocular ischaemia and orbital inflammation associated with systemic lupus erythematosus. Br J Ophthalmol 2002; 86: 474-475.

89 Burkhalter E. Unique presentation of systemic lupus erythematosus. Arthritis Rheum 1973; 16: 428.

90 Gray RE, Jenkins EA, Hall MA, Kanski JJ, Ansell BM. Recurrent acute proptosis in atypical systemic lupus erythematosus. Clin Rheumatol 1989; 8: 528-532.

91 Grimson BS, Simons KB. Orbital inflammation, myositis, and systemic lupus erythematosus. Arch Ophthalmol 1983; 101: 736-738.

92 Nabili S, McCarey DW, Browne B, Capell HA. A case of orbital myositis associated with rheumatoid arthritis. Ann Rheum Dis 2002; 61: 938-939.

93 Panfilio CB, Hernandez-Cossio O, Hernandez-Fustes OJ. Orbital myositis and rheumatoid arthritis: case report. Arq Neuropsiquiatr 2000; 58: 174-177.

94 Lateef A, Petri M. Biologics in the treatment of systemic lupus erythematosus. Curr Opin Rheumatol 2010; 22: 504-509.

95 Smolen JS, Emery P. Infliximab: 12 years of experience. Arthritis Res Ther 2011; 13(Suppl 1): S2.

96 Leandro MJ, Becerra-Fernandez E. B-cell therapies in established rheumatoid arthritis. Best Pract Res Clin Rheumatol 2011; 25: 535-548.

97 Dalakas MC. Review: an update on inflammatory and autoimmune myopathies. Neuropathol Appl Neurobiol 2011; 37: 226-242.

98 Kokotis P, Theodossiadis P, Bouros C, Sfikakis PP. Bilateral ocular myositis as a late complication of dermatomyositis. J Rheumatol 2005; 32: 379-381.

99 Marasini B, Cossutta R, Massarotti M. Bilateral ocular myositis as a late complication of dermatomyositis. J Rheumatol 2005; 32: 2498-2499; author reply 2499-2500.

100 Hak AE, de Paepe B, de Bleecker JL, Tak PP, de Visser M. Dermatomyositis and polymyositis: new treatment targets on the horizon. Neth J Med 2011; 69: 410-421. 